J. Perinat. Med.

7 (1979) 291

\title{
Multiphasic fetal monitoring in the high-risk pregnancies
}

\author{
Tsuyoshi Kaneoka, Makoto Aso, Hiroko Ozono, Koichi Shirakawa
}

Department of Obstetrics and Gynecology, Fukuoka University School of Medicine,
Japan

Fetal cardiotocography (CTG) and scalp blood $\mathrm{pH}$ determinations are thought to be the most reliable and popular methods of fetal monitoring in modern obstetrics. However, it would be clear that these two methods are still not complete to assure fetal wellbeing in utero. Thus, we have explored several methods, such as continuous monitoring of fetal tissue oxygen availability $\left(\mathrm{aO}_{2}\right)$, multiphasic determinations of feto-maternal blood biochemical parameters, fetal electrocardiographic and mechanocardiographic analyses, and so on, for the estimation of intrauterine fetal conditions. The purpose of this investigation was to clarify whether these multiphasic biophysical and biochemical parameters could be used for the accurate diagnosis of fetal distress, and how we could approach fetal safety limit during the perinatal period.

\section{Material and methods}

A total of 125 high-risk pregnancies, determined by scores designed by HoBEL et al [7] and Goodwin et al [6], were studied at the Fukuoka University Hospital during a two year period from January 1975 through December 1976. The bare platinum $\mathrm{pO}_{2}$ microelectrode and $\mathrm{Ag} / \mathrm{AgCl}$ anode, both of which were mountedin HEWLETT-PACKARD, spiral ECG electrodes (Fig. 1), were placed on the presenting part of the fetus during the early stage of labor, and changes in fetal $\mathrm{aO}_{2}$ were recorded on a RIKKADENKI multi-pen recorder, using a SHIN-EI polarographic bridge, POG-200, simultaneously with fetal CTG, obtained from direct

\section{Curriculum vitae}

TSUYOSHI KANEOKA, Born 1932 in Okayama, Japan. Medical degree in 1955 at the Okayama University. Trained at the Francis Delafield Hospital of Columbia-Presbyterian Medical Center and the Albert Einstein College of Medicine, New York, from 1958 to 1962. Completed the Postgraduate School of Okayama University in 1963. Director of Obs-Gyn Dept., National Fukuyama Hospital from 1963 to 1973. Associate Professor of Obstetrics and Gynecology, Fukuoka University from 1973.

fetal ECG and intra-amniotic pressure (IAP) measurements by a TorTsu System Tocograph, PCS260. Signals from fetal ECG, PCG and ultrasound transducers were monitored on an oscilloscope and stored in a TEAC data recorder, R-70A. Ultrasonic signals were passed through a $970 \mathrm{~Hz}$ band-pass filter. Fetal mechanocardiogram (MCG) was recorded on a SHIN-EI Visigraph 5L17. Fetal CTG was analyzed quantitatively using the dip area of SHELlEY and TIPTON [15], the bradycardie résiduelle of SUREAU et al [16], the bradycardia index of KOMAROMY cited by KrAUSE et al [10], the lag time of MYERS et al [12], the prediction index of APGAR score by SCHIFRIN and DAME [14], and the index of fetal welfare by Tipton and Shelley [17]. Determinations of 


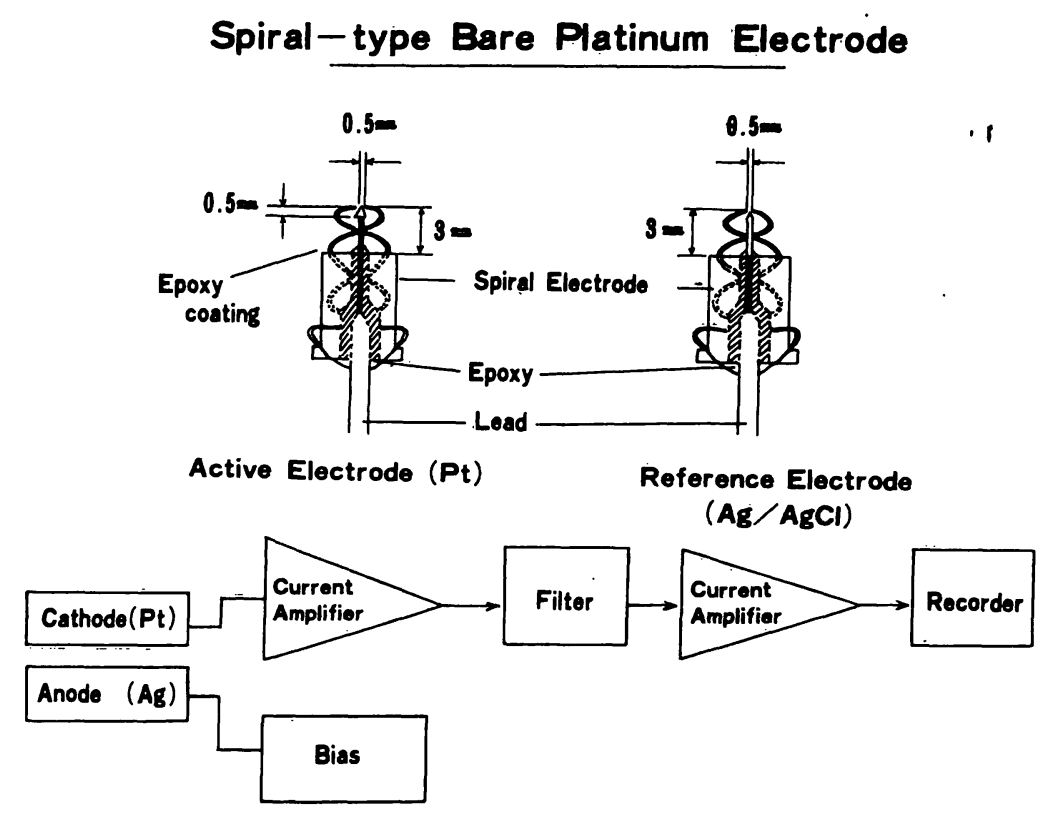

Fig. 1. The bare platinum polarographic electrode (cathode) and silver/silver chloride anode, mounted on the spiraltype fetal ECG electrodes (HEWLETT-PACKARD 1513A), and the block diagram of polarographic bridge used for the measurement of fetal tissue oxygen availability $\left(\mathrm{aO}_{2}\right)$.

$\mathrm{Po}_{2}, \mathrm{Pco}_{2}, \mathrm{pH}$, Buffer Base and Base Deficit were made using a Radiometer bloodgas analyzer on maternal peripheral venous, fetal scalp, umbilical venous and arterial, and neonatal central venous blood. Concentrations of glucose, lactate, pyruvate, ATP, glycerol, acetoacetate, $\beta-\mathrm{OH}$ butyrate, etc. were assayed on this blood using enzymatic analytical methods by BergmeYer [3]. Plasma concentrations of FFA and inorganic $P$ were measured by WAKO-JUNYAKU KIT and FISKESubBARow method, respectively. Feto-maternal excess lactate $\left(\Delta \mathrm{XL}_{\mathbf{f}-\mathrm{m}}\right)$ was calculated from maternal lactate $\left(\mathrm{L}_{\mathrm{m}}\right)$, pyruvate $\left(\mathrm{P}_{\mathrm{m}}\right)$, fetal lactate $\left(\mathrm{L}_{\mathrm{f}}\right)$ and pyruvate $\left(\mathrm{P}_{\mathrm{f}}\right)$ values, using an equation of

$$
\Delta X L=\left(L_{f}-L_{m}\right)-\left[\left(P_{f}-P_{m}\right) \frac{L_{m}}{P_{m}}\right]
$$

Statistical analyses were made by a computer, FACOM 230-25.

\section{Results}

\subsection{Fetal tissue oxygen availability}

In 1963, we [9] found that polarographic measurements of fetal $\mathrm{aO}_{2}$ would be quite valuable to detect the intrauterine environment in the canine fetus during experimentally produced hypoxia. In this clinical study, a stable polarographic current was again obtained 10 to 15 minutes after the insertion of electrodes, with a negligible drift and a minimal recidual current. The following results were obtained.

(1) Fetal scalp $\mathrm{pO}_{2}$ fluctuaged continuously probably due to local $\mathrm{pO}_{2}$ changes. However, relatively high values in mean $\mathrm{aO}_{2}$ were maintained until the terminal stage of labor in uncomplicated deliveries. Maternal $100 \%$ oxygen administration resulted in an increase in fetal $\mathrm{aO}_{2}$ of a maximal value of 2 to 3 times more than the resting level after 2 to 3 minutes of lag time. Fetal scalp $\mathrm{aO}_{2}$ increased simultaneously with uterine contractions at the early stage of labor, reaching the maximal level at the peak of contractions and the minimal after contractions. When IAP increased to an average value of $35 \pm 10$ torr, the fetal $\mathrm{aO}_{2}$ became biphasic, demonstrating a marked decrease after contractions. Occasionaly, a mirror image could be observed against IAP tracings. (2) When IAP reached to the average level of $70 \pm 20$ torr, fetal $\mathrm{aO}_{2}$ decreased remarkably, usually showing a consistently low level throughout the late stage of labor. Tab. I indicates the relationship between a time interval from a marked $\mathrm{aO}_{2}$ decrease to delivery and umbilical arterial blood biochemical 
Tab. I. Relationships between time duration from a marked decrease in fetal tissue oxygen availability to delivery, and cardiotocographic and umbilical arterial blood biochemical values.

Time duration from a marked decrease in fetal tissue $\mathrm{aO}_{2}$ to delivery.

$<10$ Min.

27
10-20 Min. 28
$20-30 \mathrm{Min}$. 16
K 30 Min.

12

Numbers $($ Total $=83)$

(Mean \pm S.D.)

(Mean \pm S.D.)

(Mean \pm S.D.)

(Mean \pm S.D.)

APGAR score

(Cardiotocographic Values)

9.4

0.9

8.4

2.0

8.2

2.0

7.3

3.0

Dip Area

Bradycardie résiduelle

KOMAROMY Index

SCHIFRIN-DAME Index

TIPTON-SHELLEY IndeX

$\begin{array}{cccccccc}153.8 & 115.2 & 302.2 & 156.4 & 481.2 & 236.7 & 356.1 & 202.9 \\ 14.8 & 27.8 & 35.8 & 32.7 & 85.5 & 58.4 & 55.3 & 58.6 \\ 0.33 & 0.33 & 0.69 & 0.98 & 0.93 & 0.40 & 0.94 & 0.59 \\ 9.0 & 1.6 & 8.2 & 1.9 & 6.4 & 2.4 & 6.3 & 2.6 \\ 8.4 & 1.3 & 7.0 & 1.7 & 5.5 & 1.2 & 6.2 & 2.4\end{array}$

(Umbilical Arterial Blood Values)

\begin{tabular}{lllllllllr}
{$\left[\mathrm{H}^{+}\right]$} & $(\mathrm{nM} / \mathrm{L})$ & 51.6 & 6.5 & 59.3 & 9.4 & 68.6 & 11.3 & 66.9 & 17.8 \\
Base Deficit & $(\mathrm{mEq} / \mathrm{L})$ & 10.2 & 3.0 & 13.2 & 3.4 & 15.1 & 4.5 & 14.3 & 2.8 \\
$\mathrm{PcO}_{2}$ & $($ torr & 39.1 & 8.6 & 41.5 & 9.1 & 44.7 & 11.0 & 48.4 & 22.0 \\
$\mathrm{Lactate}$ & $(\mathrm{mM} / \mathrm{L})$ & 1.90 & 0.51 & 2.62 & 0.77 & 3.38 & 1.24 & 2.75 & 1.03 \\
$\Delta \mathrm{XL}_{\mathrm{f}-\mathrm{m}}$ & $\mathrm{M} / \mathrm{L})$ & 0.60 & 0.69 & 0.99 & 0.52 & 1.07 & 0.64 & 1.23 & 0.66 \\
Glycerol & $(\mathrm{mM} / \mathrm{L})$ & 0.15 & 0.09 & 0.20 & 0.09 & 0.21 & 0.11 & 0.19 & 0.07 \\
inorganic $\mathrm{P}$ & $(\mathrm{mM} / \mathrm{L})$ & 1.44 & 0.16 & 1.52 & 0.27 & 1.65 & 0.23 & 1.58 & 0.32 \\
\hline
\end{tabular}

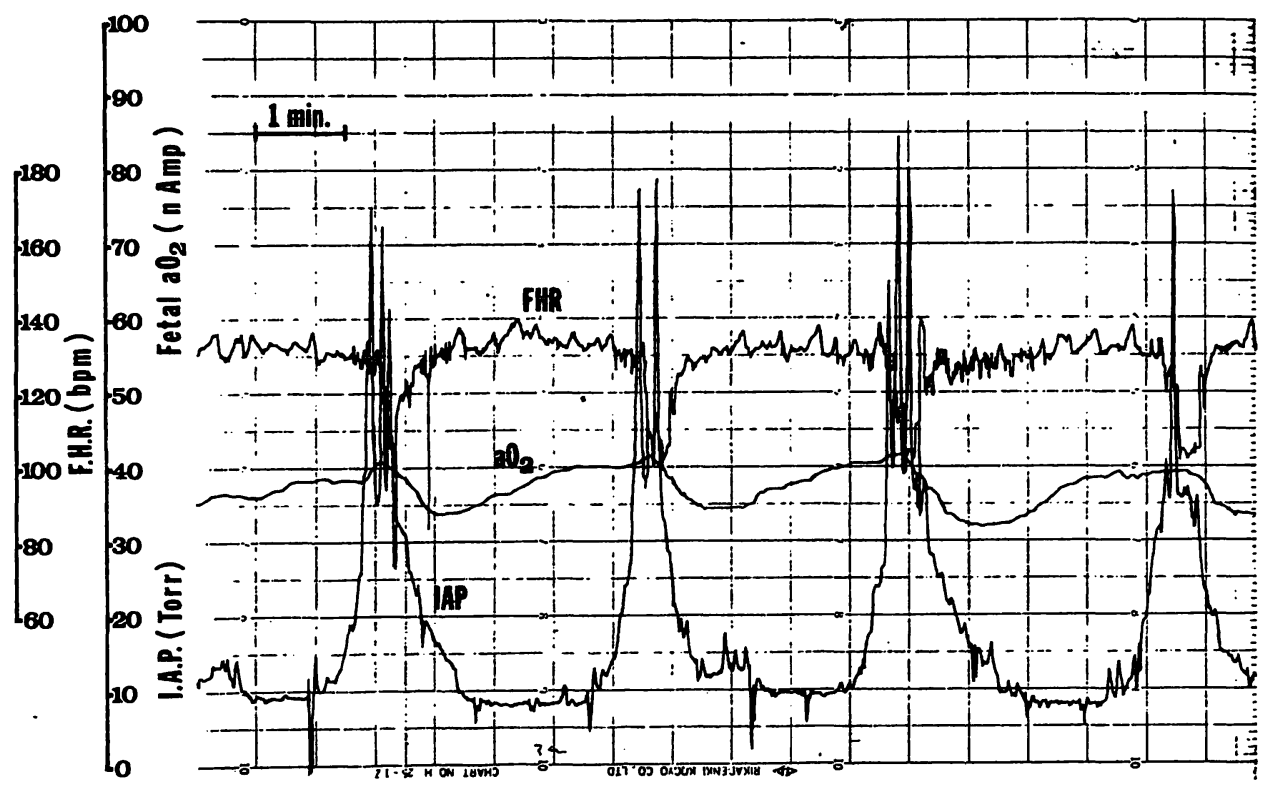

Fig. 2. Simultaneous recording of CTG and fetal tissue $\mathrm{aO}_{2}$. FHR demonstrates an early deceleration, while fetal tissue $\mathrm{aO}_{2}$ demonstrates an increase together with uterine contractions.

values. When the $\mathrm{aO}_{2}$ decrease to delivery time prolonged more than 20 to 30 minutes, abnormal value were usually found in CTG, APGAR, and biochemical parameters. (3) Using simultaneous recordings of CTG and fetal $\mathrm{aO}_{2}, \mathrm{CTG}$ tracings could be clearly classified in three categories: (a) early, vagal, or head compression type deceleration characterized by a deceleration with a slight change in fetal $\mathrm{aO}_{2}$ (Fig. 2), late, anoxic or uteroplacental insufficiency type deceleration charac- 


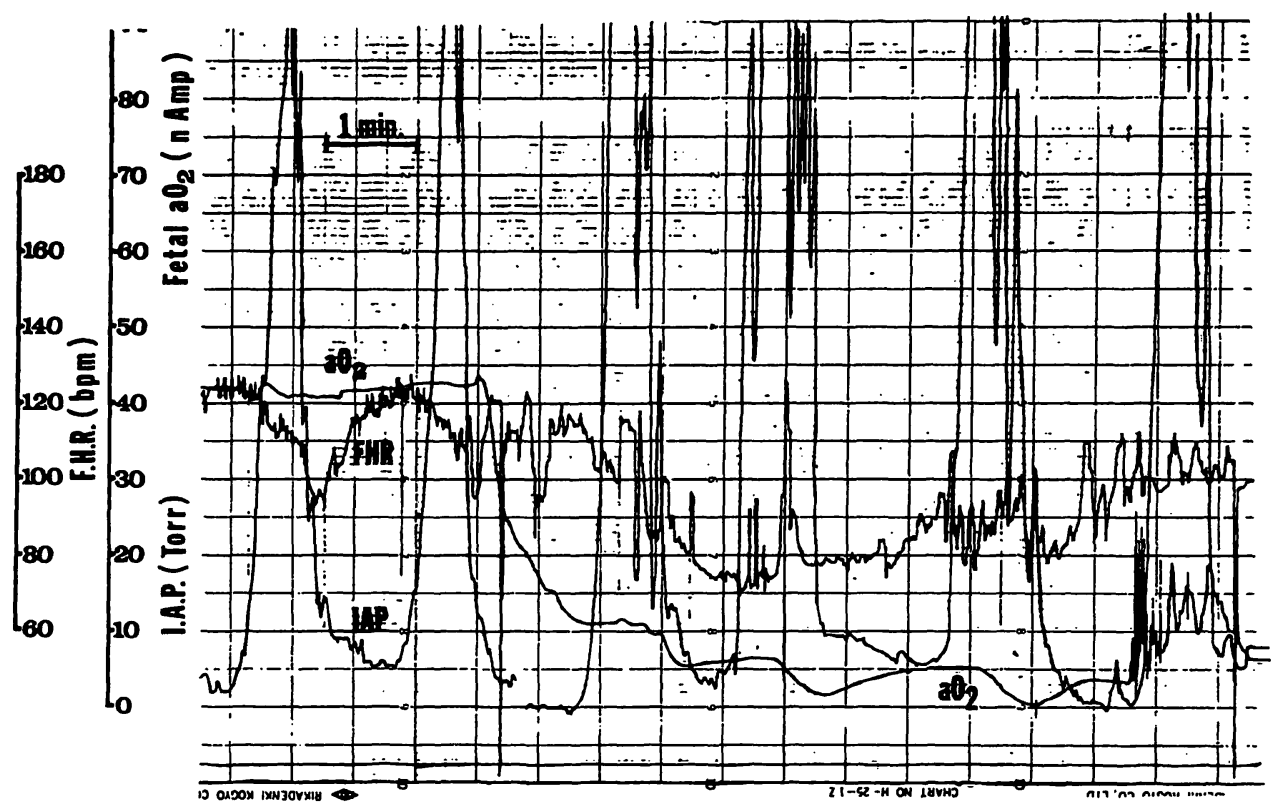

Fig. 3. A remarkable decrease in fetal tissue $\mathrm{aO}_{2}$ observed at the terminal stage of labor, followed by a late deceleration and baseline bradycardia.

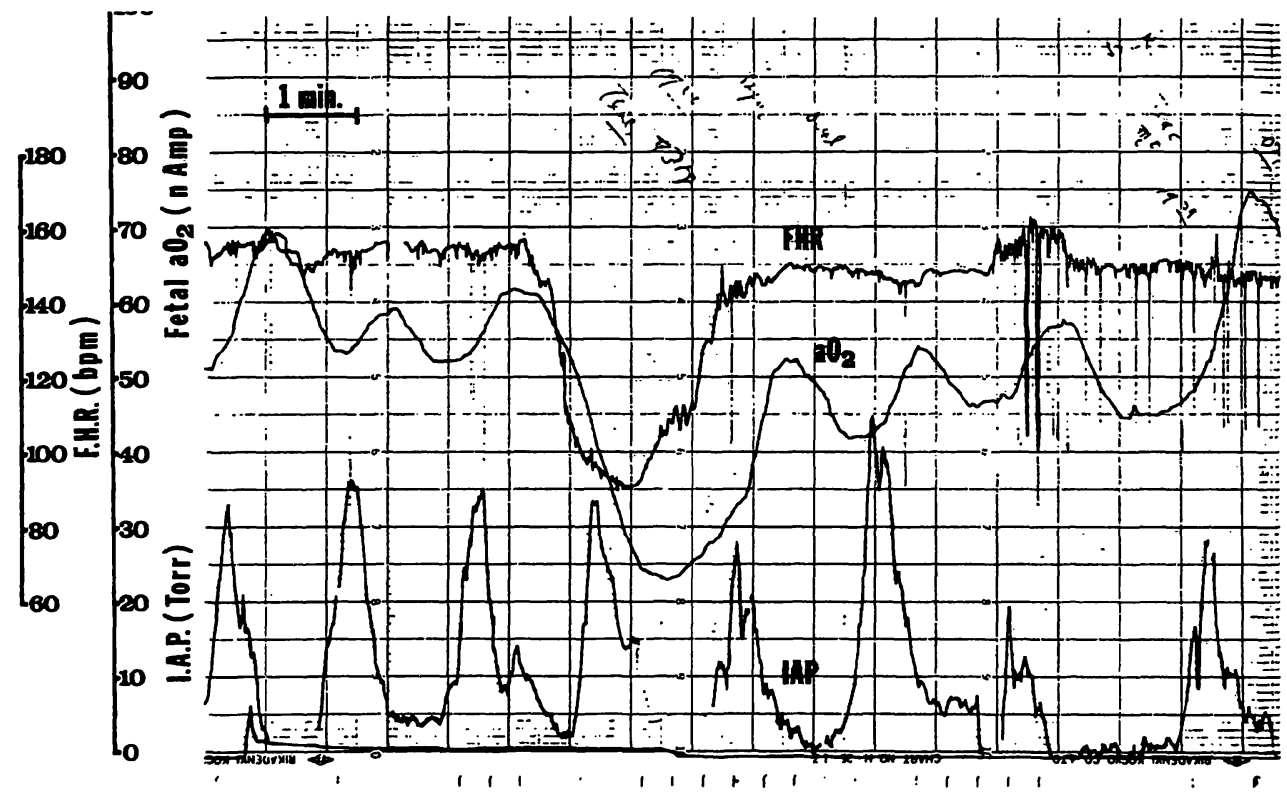

Fig. 4. Simultaneous decrease in fetal $\mathrm{aO}_{2}$ and FHR: Variable deceleration.

terized by a secondary deceleration following an initial remarkable fall in fetal $\mathrm{aO}_{2}$ (Fig. 3) and (c) variable, hemodynamic, or cord compression type deceleration characterized by a simultaneous decrease in FHR and fetal $\mathrm{aO}_{2}$ (Fig. 4).

(5) Even in the presence of fetal acidemia, maternal $\mathrm{O}_{2}$ administration could usually recover both FHR and fetal $\mathrm{aO}_{2}$, suggesting that FHR would be more dependent on fetal blood $\mathrm{pO}_{2}$ than blood $\mathrm{pH}$.

\subsection{CTG quantitative values and blood biochemi- cal values}

As shown in Tab. II, APGAR scores correlated'significantly ( $p<0.01)$ with both CTG quantitative values, such as bradycardie résiduelle, ScHIFRINDAME index, TIPTON-Shelley index, dip area, KOMAROMY index, and lag time and several umbilical arterial blood biochemical parameters, 
Tab. II. Correlation matrix between APGAR scores, cardiotocographic quantitative values, and umbilical arterial blood biochemical values.

\begin{tabular}{|c|c|c|c|c|c|c|c|}
\hline APGAR score & $\begin{array}{l}\text { APGAR } \\
\text { score } \\
- \\
\end{array}$ & $\begin{array}{l}\begin{array}{l}\text { Dip } \\
\text { area }\end{array} \\
-0.359 \\
\end{array}$ & $\begin{array}{l}\text { Brady cardie } \\
\text { résiduelle } \\
-0.561 \\
\end{array}$ & $\begin{array}{l}\text { KOMAROMY } \\
\text { Index } \\
-0.264 \\
\end{array}$ & $\begin{array}{l}\text { Lag } \\
\text { Time } \\
-0.241 \\
\end{array}$ & $\begin{array}{c}\text { SCHIFRIN- } \\
\text { DAME I. } \\
0.541 \\
\end{array}$ & $\begin{array}{l}\text { TIPTON- } \\
\text { SHELLEY I. } \\
-0.466 \\
\end{array}$ \\
\hline $\begin{array}{l}{\left[\mathrm{H}^{+}\right]} \\
\Delta\left[\mathrm{H}^{+}\right]_{f-m} \\
\text { Base Deficit } \\
\text { inorganic } \mathrm{P} \\
\Delta \mathrm{XL} \text { f-m } \\
\Delta \mathrm{PcO}{ }_{2} \mathrm{f}-\mathrm{m} \\
\text { Buffer Base } \\
\text { Glycerol } \\
\text { Lactate }\end{array}$ & $\begin{array}{r}-0.547 \\
-0.538 \\
-0.455 \\
-0.426 \\
-0.404 \\
-0.381 \\
0.332 \\
-0.250 \\
-0.168\end{array}$ & $\begin{array}{r}0.347 \\
0.360 \\
0.287 \\
0.116 \\
0.471 \\
0.286 \\
-0.513 \\
0.210 \\
0.546\end{array}$ & $\begin{array}{r}0.293 \\
0.336 \\
0.191 \\
0.547 \\
0.359 \\
0.295 \\
-0.182 \\
0.294 \\
0.241\end{array}$ & $\begin{array}{r}0.358 \\
0.616 \\
0.365 \\
0.277 \\
0.381 \\
0.541 \\
-0.391 \\
0.113 \\
0.403\end{array}$ & $\begin{array}{r}0.329 \\
0.373 \\
0.319 \\
0.195 \\
0.387 \\
0.306 \\
-0.406 \\
0.091 \\
0.485\end{array}$ & $\begin{array}{r}-0.468 \\
0.499 \\
-0.231 \\
-0.269 \\
-0.363 \\
-0.432 \\
0.328 \\
-0.126 \\
-0.311\end{array}$ & $\begin{array}{r}-0.456 \\
0.544 \\
-0.201 \\
-0.279 \\
-0.446 \\
-0.340 \\
0.372 \\
-0.098 \\
-0.378\end{array}$ \\
\hline
\end{tabular}

$(n=125(d . f=123), \alpha=0.05$ correlation limit: $0.176, \alpha=0.01$ correlation limit: 0.230$)$

Tab. III. Correlation matrix between APGAR scores, cardiotocographic quantitative values, and postnatal 15-minute central venous blood biochemical values.

\begin{tabular}{|c|c|c|c|c|c|c|c|}
\hline APGAR score & $\begin{array}{l}\text { APGAR } \\
\text { score } \\
- \\
\end{array}$ & $\begin{array}{l}\text { Dip } \\
\text { area } \\
-0.588 \\
\end{array}$ & $\begin{array}{l}\text { Bradycardie } \\
\text { résiduelle } \\
-0.594 \\
\end{array}$ & $\begin{array}{l}\text { KOMAROMY } \\
\text { Index } \\
-0.701 \\
\end{array}$ & $\begin{array}{l}\text { Lag } \\
\text { Time } \\
-0.284 \\
\end{array}$ & $\begin{array}{c}\text { SCHIFRIN- } \\
\text { DAME I. } \\
0.611 \\
\end{array}$ & $\begin{array}{l}\text { TIPTON- } \\
\text { SHELLEY I. } \\
0.613\end{array}$ \\
\hline $\begin{array}{l}{\left[\mathrm{H}^{+}\right]} \\
\text {Lactate } \\
\Delta \mathrm{XL}_{\mathrm{f}-\mathrm{m}} \\
\mathrm{PCO}_{2} \\
\text { inorganic } \mathrm{P} \\
\mathrm{L} / \mathrm{P} \text { ratio } \\
{\left[\mathrm{HCO}_{3}\right]} \\
\text { Glycerol } \\
\text { Glucose }\end{array}$ & $\begin{array}{r}-0.784 \\
-0.658 \\
-0.630 \\
-0.614 \\
-0.613 \\
-0.569 \\
0.489 \\
-0.423 \\
-0.419\end{array}$ & $\begin{array}{r}0.717 \\
0.647 \\
0.519 \\
0.545 \\
0.496 \\
0.643 \\
-0.397 \\
0.396 \\
0.269\end{array}$ & $\begin{array}{r}0.741 \\
0.639 \\
0.536 \\
0.586 \\
0.540 \\
0.675 \\
-0.356 \\
0.360 \\
0.239\end{array}$ & $\begin{array}{r}0.602 \\
0.699 \\
0.560 \\
0.433 \\
0.497 \\
0.475 \\
-0.462 \\
0.393 \\
0.322\end{array}$ & $\begin{array}{r}0.267 \\
0.375 \\
0.184 \\
0.114 \\
0.322 \\
0.092 \\
-0.337 \\
0.185 \\
0.146\end{array}$ & $\begin{array}{r}-0.565 \\
-0.591 \\
-0.466 \\
-0.425 \\
-0.405 \\
-0.407 \\
0.431 \\
-0.326 \\
-0.225\end{array}$ & $\begin{array}{r}-0.578 \\
-0.642 \\
-0.534 \\
-0.366 \\
-0.355 \\
-0.410 \\
0.516 \\
-0.363 \\
-0.315\end{array}$ \\
\hline
\end{tabular}

$(n=70(d . f=69), \alpha=0.05$ correlation limit: $0.235, \alpha=0.01$ correlation limit: $(0.306)$

such as $\left[\mathrm{H}^{+}\right]$Base Deficit, inorganic $\mathrm{P}, \Delta \mathrm{XL}_{\mathbf{f}-\mathrm{m}}$, Buffer Base and glycerol concentrations. Significant correlations were also evident between CTG quantitative values and some umbilical blood values. However, as shown in Tab. III, neonatal 15-minute central venous blood biochemical values were better correlated with APGAR scores and CTG values than umbilical arterial blood values. Thus, it was concluded that the biological stress of labor would initially relfect on CTG quan. titative values during the intrapartum period, subsequently on APGAR scores at birth, and finally on neonatal 15-minuite blood biochemical values after birth. These correlations were lost at
30- and 60-minute blood biochemical values. Tab. IV indicates mean values of CTG umbilical arterial blood biochemical and postnatal 15-minute central venous blood biochemical values in these high-risk pregnancies and those APGAR scores more than 8 .

\subsection{Fetal electrocardiogram and mechanocardio- gram}

By continuous monitoring of direct-lead fetal ECG configurations, vagal deceleration could be differentiated from other dips by a suppression or disappearance of $P$ waves (Fig. 5). However, ST/T 
Tab. IV. Cardiotocographic, umbilical arterial blood biochemical, and postnatal 15-minute central venous blood biochemical values.

i

\begin{tabular}{|c|c|c|c|c|c|c|c|}
\hline & & \multicolumn{3}{|c|}{ In all populations $(n=91)$} & \multicolumn{3}{|c|}{ In those APGAR scores $\geqslant 8(n=55)$} \\
\hline & & Mean & S.D. & $\begin{array}{l}\text { Confidence } \\
\text { Limit }\end{array}$ & Mean & S.D. & $\begin{array}{l}\text { Confidence } \\
\text { Limit }\end{array}$ \\
\hline \multicolumn{8}{|c|}{ (Cardiotocographic Values) } \\
\hline Dip Area & & 349.7 & 391.3 & 443 & 169.9 & 104.3 & 219 \\
\hline Bradycardie & & 66.1 & 128.1 & 97 & 18.0 & 25.2 & 30 \\
\hline KOMAROM & & 0.78 & 0.86 & 0.98 & 0.35 & 0.28 & 0.48 \\
\hline Lag Time & (sec.) & 19.8 & 23.6 & 25.4 & 5.6 & 12.2 & 11.3 \\
\hline SCHIFRIN-I & $\operatorname{dex}$ & 7.7 & 2.5 & 7.1 & 9.4 & 1.0 & 8.9 \\
\hline TIPTON-SH & ndex & 7.0 & 2.4 & 6.5 & 8.6 & 1.1 & 8.1 \\
\hline \multicolumn{8}{|c|}{ (Umbilical Arterial Values) } \\
\hline$\left[\mathrm{H}^{+}\right]$ & $(\mathrm{nM} / \mathrm{L})$ & 59.6 & 11.9 & 62.1 & 51.6 & 6.0 & 54.3 \\
\hline Lactate & $(\mathrm{mM} / \mathrm{L})$ & 2.33 & 0.96 & 2.52 & 1.90 & 0.47 & 0.70 \\
\hline$\Delta \mathrm{XL}_{\mathrm{f}-\mathrm{m}}$ & $(\mathrm{mM} / \mathrm{L})$ & 0.81 & 0.70 & 0.96 & 0.50 & 0.43 & 0.70 \\
\hline \multicolumn{8}{|c|}{ (Postnatal 15-minute Values) } \\
\hline$\left[\mathrm{H}^{+}\right]$ & $(\mathrm{nM} / \mathrm{L})$ & 64.7 & 25.0 & 69.9 & 51.4 & 10.8 & 56.5 \\
\hline Lactate & $(\mathrm{mM} / \mathrm{L})$ & 3.37 & 1.72 & 3.73 & 2.74 & 0.63 & 3.04 \\
\hline$\Delta \mathrm{XL}_{\mathrm{f}-\mathrm{m}}$ & $(\mathrm{mM} / \mathrm{L})$ & 1.75 & 1.55 & 2.07 & 1.27 & 0.64 & 1.56 \\
\hline $\mathrm{PCO}_{2}$ & (torr) & 43.2 & 26.7 & 48.8 & 34.2 & 8.2 & 38.0 \\
\hline inorganic $P$ & $(\mathrm{mM} / \mathrm{L})$ & 1.56 & 0.31 & 1.64 & 1.45 & 0.22 & 1.56 \\
\hline $\mathrm{L} / \mathrm{P}$ ratio & (mol/mol) & 27.3 & 16.0 & 30.6 & 26.2 & 5.4 & 28.7 \\
\hline$\left[\mathrm{HCO}_{3}\right]$ & $(\mathrm{mEq} / \mathrm{L})$ & 15.0 & 4.0 & 14.1 & 15.9 & 3.1 & 14.4 \\
\hline
\end{tabular}

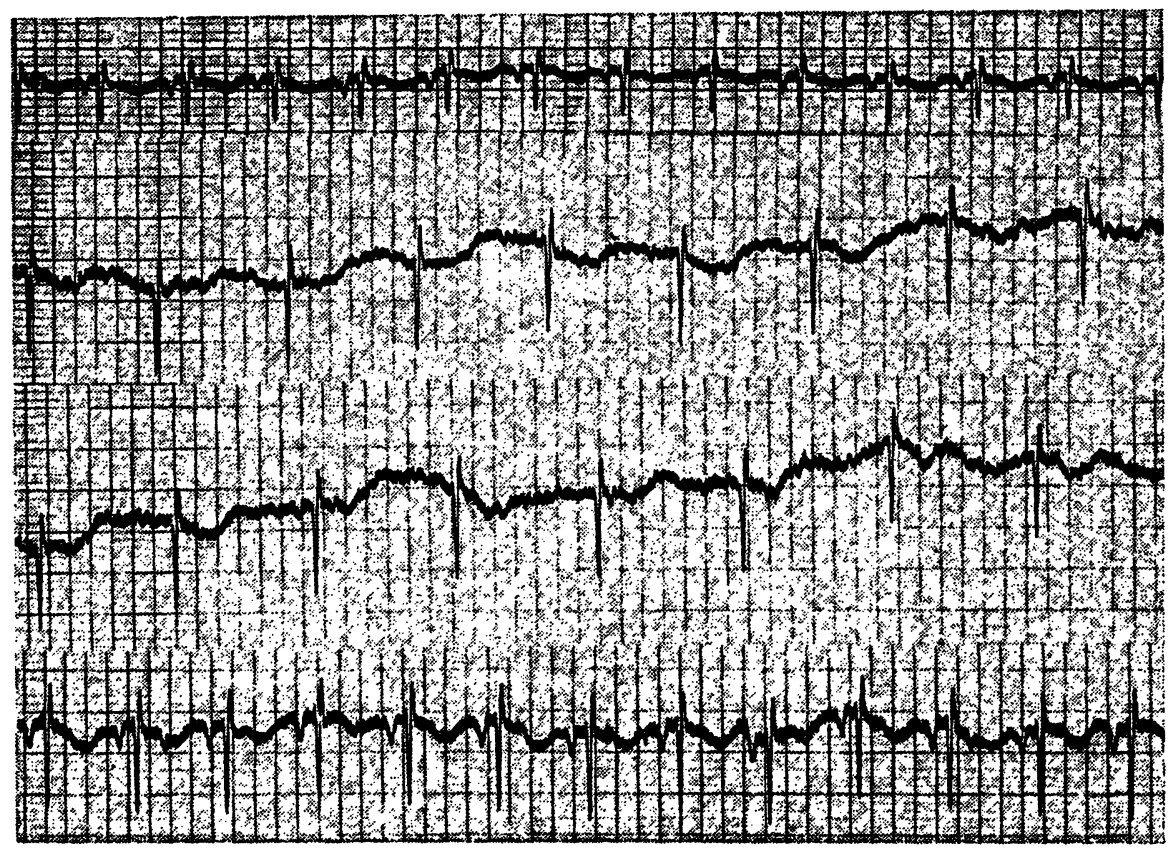

Fig. 5. Suppression or disappearance of $P$ waves observed during an early or vagal deceleration. 
changes were not always associated with anoxic FHR or $\mathrm{aO}_{2}$ changes. In MCG recordings (Fig. 6), $\mathrm{Q}-\mathrm{S}_{2}$ interval represents the total electro-mechanical systole, $\mathrm{Q}-\mathrm{A}_{0}$ interval means the pre-ejection period (PEP) and $Q-S_{2}-Q-A_{0}$ signifies the left ventricular ejection time (LVET). In our investigation, $\mathrm{Q}-\mathrm{S}_{2}$ was found to be highly $\mathrm{FHR}(\mathrm{X})$ dependent $(r=-0.603)$, and calculated to be $\mathrm{Y}(\mathrm{msec})=-0.851 \mathrm{X}+(354.2 \pm 33.54) . \mathrm{Q}-\mathrm{A}_{0}$ was also correlated with FHR ( $\mathrm{r}=-0.273)$, and calculated to be $Y=-0.132 X+(89.87 \pm 13.85)$. Although $\mathrm{Q}-\mathrm{S}_{2}$ and $\mathrm{Q}-\mathrm{A}_{0}$ were said to be shortened by fetal sympathetic response with cathecolamine secretion and prolonged by anoxic myocardial damage [5], these parameters were changed inconsistently in a minute-to-minute interval in fetal distress. As shown in Tab. V, when the incidence of $\mathrm{Q}-\mathrm{A}_{\mathbf{0}}$ interval abnormalities (either

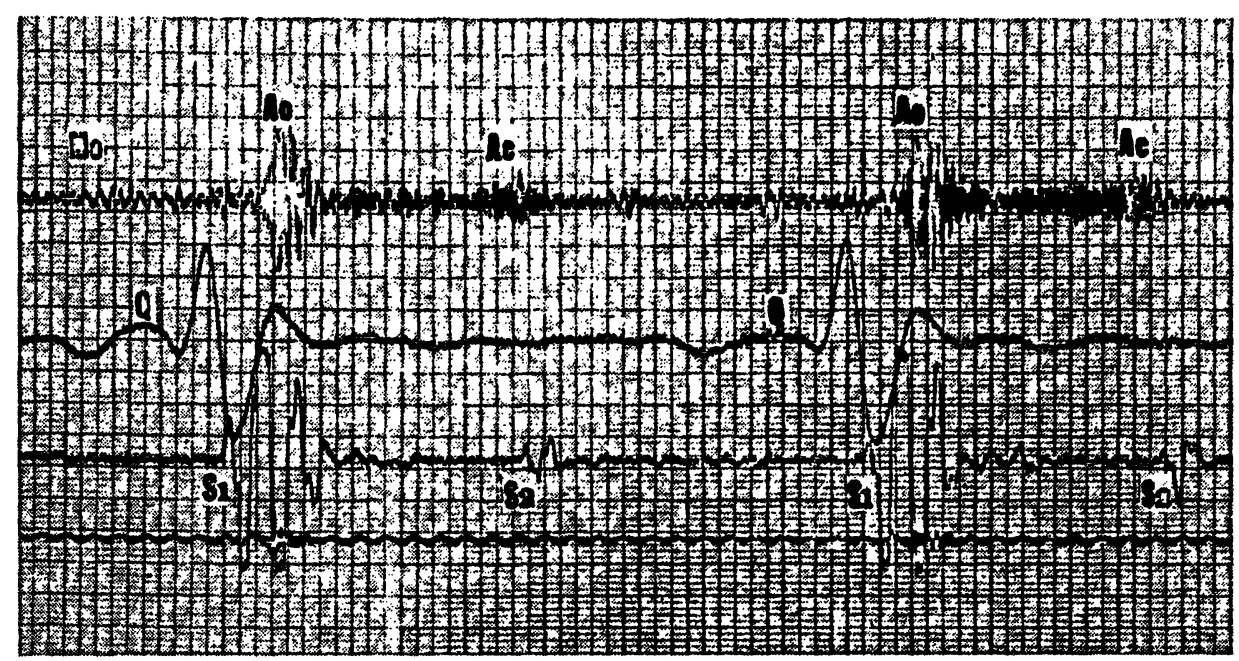

Fig. 6. Fetal mechanocardiogram, consisted of fetal ultrasonogram, electrocardiogram, and phonocardiogram. Line interval was $10 \mathrm{msec}$.

Tab. V. Relationships between the incidence of Q-Ao (Pre-Ejection Period) abnormalities, and cardiotocographic and umbilical arterial blood biochemical values.

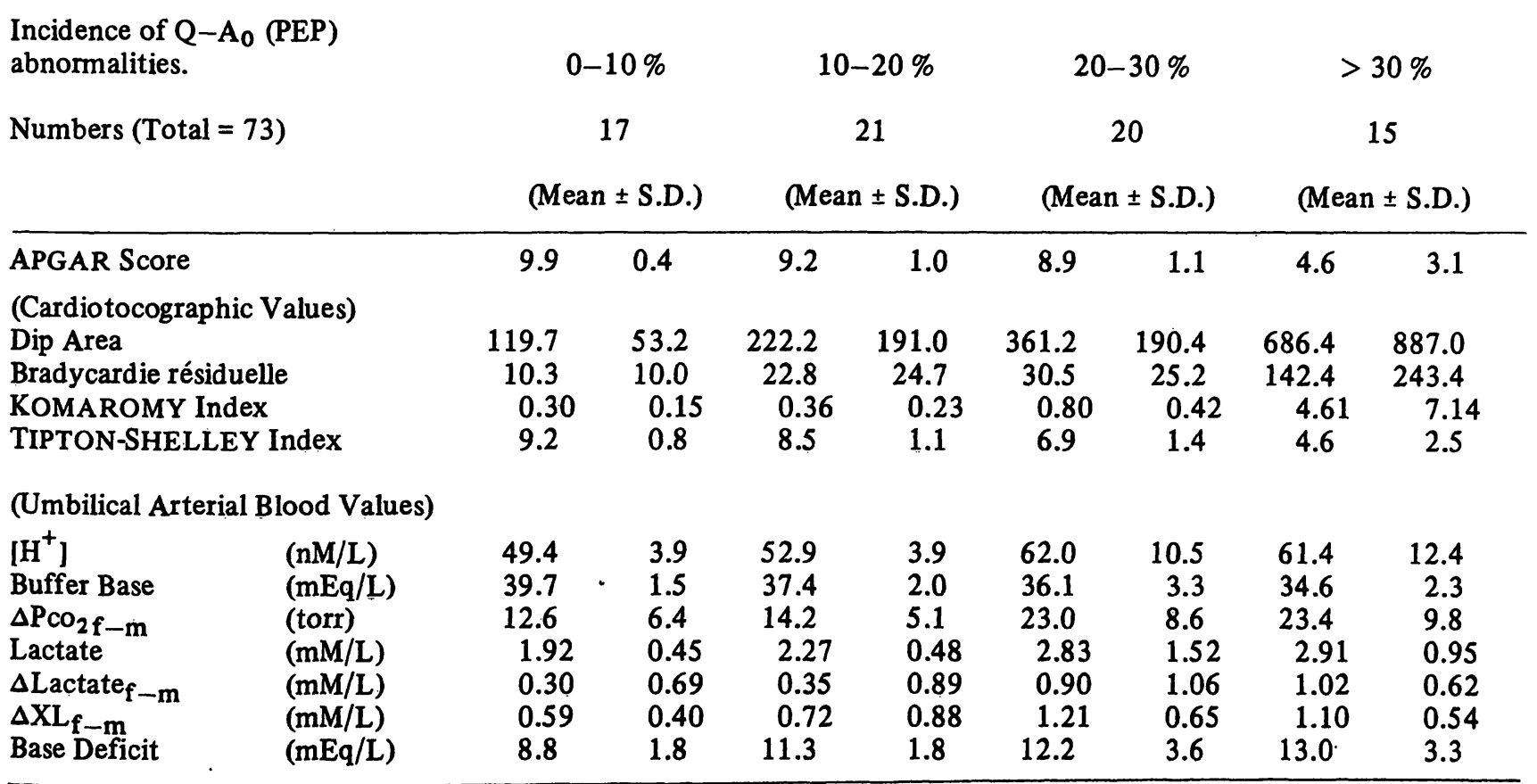


shortening or prolongation from normal range stated above) in a unit time at the terminal stage of labor exceeded more than 20 to $30 \%$, abnormal values were found in APGAR scores, CTG quantitative values and blood biochemical profilings. Thus, it was concluded that continuous monitoring of MCG might be beneficial in accurate diagnosis of fetal distress in utero.

\section{Comment}

Although CTG recordings are widely used in modern obstetrics, their interpretations are still confusing. Since each fetus has a different amount of reserve capacities as well as a different function of sympathetic, endocrine and other defense mechanism against the intrapartum stress, multiphasic, not merely single, monitoring of the fetus should be emphasized. Measurements of fetal tissue $\mathrm{aO}_{2}$ were made experimentally in 1963 by us [9], and clinically by ALthaBe et al [1], WALKER et al $[18,19]$, and FRANKE et al [4]. Recently HuCH et al [8] reported absolute $\mathrm{Po}_{2}$ changes during labor, using a heated transcutaneous CLARK-type electrode. However, our electrodes in this investigation were thought to be simple and effective for better understanding of fetal CTG and cardiorespiratory physiology.

APGAR scores, originated by the late APGAR [2] in 1953 to evaluate the neonatal conditions at birth, were known to be not always well correlated with umbilical arterial blood biochemical values. Since we have found in this study that APGAR scores were more correlated with neonatal 15-minute biochemical profilings than umbilical blood values, the existence of a time factor in these biochemical parameters might be suggested.

Although morphologic changes in fetal ECG were less promising, fetal MCG analyses, described by Murata et al [11], Organ et al [13], and GooduIn et al [5], were thought to be useful for an accurate diagnosis of fetal distress. Further technical development would be necessary for continuous monitoring of fetal mechanocardiogram.
Summary

The purpose of this study was to monitor the fetus during labor by multiphasic biochemical and biophysical parameters, such as fetal tissue oxygen availability, cardiotocographic quantitative values, maternofetal blood biochemical values, electrocardiogram, mechanocardiogram, etc., for the accurate diagnosis of fetal distress. A total of 125 high-risk pregnancies were investigated. The polarographic electrodes, mounted on spiral fetal ECG electrodes, were inserted into the presenting part of the fetus at the early stage of labor. Direct cardiotocographic recordings were analyzed by quantitative measures designed by others. Fetal mechanocardiogram was obtained by ECG, PCG and ultrasound transducers and stored for analyses. Determinations of blood gas and acid-base balance values were made on maternal, fetal, umbilical and neonatal blood. Enzymatic analyses of metabolic substrates were also made on these blood samples. The following results were obtained:

(1) By continuous monitoring of fetal tissue oxygen availability, fetal tissue $\mathrm{aO}_{2}$ was thought to be a good index to understand changes in CTG and fetal respiratory physiology. Although no remarkable decrease in $\mathrm{aO}_{2}$ was observed in non-stressed optimal deliveries, marked $\mathrm{aO}_{2}$ decrease of more than $\mathbf{2 0}$ to $\mathbf{3 0}$ minutes duration in complicated deliveries resulted in abnormal values in cardiotocographic, APGAR and umbilical arterial blood biochemical parameters (Tab. I).
(2) APGAR scores were found to be significantly correlated with CTG quantitative values, such as the bradycardie résiduelle, SCHIFRIN-DAME index, TIPTONSHELLEY index, the dip area, KOMAROMY index, and the lag time, and also with umbilical arterial blood biochemical values, such as $\left[\mathrm{H}^{+}\right]$, Base Deficit, inorganic $P$, Buffer Base and glycerol concentrations (Tab. II). However, better correlations were found between APGAR scores and neonatal 15-minute blood biochemical profilings, consisting of $\left[\mathrm{H}^{+}\right]$, lactate, $\Delta \mathrm{XL}_{\mathbf{f}}=\mathrm{m}, \mathrm{P}_{\mathrm{co} 2}$, inorganic $\mathrm{P}$, lactate/pyruvate ratio, $\left[\mathrm{HCO}_{3}^{-}\right]$, glycerol and glucose concentrations (Tab. III), suggesting time factors in these biochemical parameters.

(3) In fetal MCG, normal range in the fetal pre-ejection time $\left(\mathbf{Q}-\mathbf{A}_{0}\right)$ was calculated to be $\mathrm{Y}(\mathrm{msec})=-0.132 \mathrm{X}$ $+(89.87 \pm 13.85)$ to FHR $(X)$. When the incidence of Q-A $\mathbf{A}_{0}$ abnormalities, eigher shortening or prolongation from ranges stated above, in a unit time exceeded more than 20 to $30 \%$ at the terminal stage of labor, abnormal values were observed in CTG quantitative; APGAR and blood biochemical parameters (Tab. V). The total electromechanical systole $\left(Q-S_{2}\right)$ was calculated to be $Y=$ $-0.851 \mathrm{X}+(354.2 \pm 33.54)$, which was more FHR dependent than $Q-A_{0}$.

Since CTG is merely one parameter of fetal responses against the stress of labor and each fetus has a different degree of reserve capacities and defense mechanisms 
against the stress, the necessities of multiphasic fetal monitoring in high-risk pregnancies were discussed and emphasized. Continuous monitoring of fetal tissue oxygen level and mechanocardiographic analysis of the fetal heart, together with blood biochemical measurements, would be promising for the accurate diagnosis of fetal wellbeings in utero.

Keywords: Acidosis, anoxia, blood biochemistry, blood gas, cardiotocography, electrocardiography, fetus, fetal monitoring, mechanocardiography, polarography.

\section{Zusammenfassung}

Mehrgleisige fetale Intensiviberwachung bei Risikoschwangerschaften.

Ziel dieser Untersuchung war die subpartuale Intensivüberwachung des Feten, wobei verschiedene biochemische und biophysikalische Parameter berücksichtigt wurden, wie z. B. die Sauerstoffversorgung des fetalen Gewebes, cardiotokographische Größen, biochemische Analysen im maternalen und fetalen Blut, Elektrocardiogramm, Mechanocardiogramm usw.; es kam uns letztendlich auf eine exakte Diagnose des fetalen Stresszustandes an.

Wir untersuchten insgesamt 125 Risikoschwangerschaften. Die polarographischen Elektroden wurden auf spiralige EKG-Elektroden montiert und bei Geburtsbeginn dem vorangehenden Teil des Kindes aufgesetzt. Die intrauterin abgeleiteten cardiotokographischen Größen wurden quantitativ analysiert. Über das ECG, das PCG und die Ultraschallaufzeichnungen ließ sich ein Mechanocardiogramm erstellen; die Werte wurden für die Analyse gespeichert. Blutgaswerte und Säure-Basen-Status bestimmten wir im mütterlichen und fetalen Blut, ebenso im Nabelschnurblut sowie im Blut des Neugeborenen. Alle Blutproben wurden auch enzymatisch analy siert.

Wir erhielten folgende Ergebnisse:

1. Die kontinuierliche Überwachung der Sauerstoffversorgung des fetalen Gewebes ließ deutlich werden, daß $\mathrm{O}_{2}$-Gehalt des fetalen arteriellen Blutes und Veränderungen im CTG sowie atemphysiologische Reaktionen in Abhängigkeit voneinander stehen. Während bei unkomplizierten Geburtsverläufen kein nennenswerter $\mathrm{O}_{2}$-Abfall im arteriellen Blut festzustellen war, traten bei schwierigen Geburten Intervalle von 20 bis 30 Minuten auf, wo der arterielle $\mathrm{O}_{2}$-Gehalt deutlich erniedrigt war. Dies führte zu Abweichungen im CTG und zu abnormen Werten bei der biochemischen Analyse des Nabelschnurblutes. Auch die APGAR-Werte zeigten eine Abhängigkeit von solchen Intervallen. (Tab. I)

2. Wir fanden, daß die APGAR-Werte signifikant korrelant waren mit abweichenden quantitativen Größen im CTG, (z. B. Bradycardie résiduelle, SCHIFRIN-DAMEIndex, TIPTON-SHELLEY-Index, Dezelerationsfläche, KOMAROMY-Index und Verzögerungszeit) sowie mit
Veränderungen der biochemischen Parameter im Nabelschnurarterienblut (z. B. $\mathrm{H}^{+}$Konzentration, Basenmangel, anorganisches Phosphat und Glyzerinkonzentration). (Tab. II)

Noch besser war die Korrelation zwischen APGAR Wert und biochemischem Blutprofil in Proben, die 15 Minuten post partum entnommen wurden. Dabei wurden berücksichtigt: $\left[\mathrm{H}^{+}\right]$, Lactat, $\Delta \mathrm{XL}_{\mathrm{f}-\mathrm{m}}, \mathrm{pCO}_{2}$, anorganisches Phosphat, Verhältnis von Lactat $\mathrm{zu}$ Pyruvat, $\left[\mathrm{HCO}_{3}^{-}\right]$, Glyzerin- und Glukose-Konzentration (Tab. III). Man muß annehmen, daß der Zeitfaktor hinsichtlich dieser biochemischen Parameter von Bedeutung ist.

3. Im Mechanocardiogramm bestimmten wir die Dauer der Anspannungsphase $\left(Q-A_{0}\right)$ und die Dauer der gesamten elektromechanischen Systole $\left(Q-S_{2}\right)$. Den Normbereich für die Anspannungsphase bestimmten wir nach der Beziehung: $Y(m s e c)=-0.132 X+$

(89.87 \pm 13.85$)$, wobei $X$ die fetale Herzfrequenz angibt. Wenn Abweichungen hinsichtlich der Dauer von Q- $\mathbf{A}_{0}$ auftraten, indem sie den oben definierten $\mathrm{Be}-$ reich über- oder auch unterschritten, und die Abweichung bei Geburtsbeendigung mehr als $2030 \%$ pro Zeiteinheit betrug, so konnten Abnormitäten im CTG sowie abweichende APGAR-Werte und Blutanalysenwerte beobachtet werden (Tab. V). Die Dauer der gesamten elektromechanischen Systole wurde nach der folgenden Gleichung berechnet: $Y(\mathrm{msec})=-0.851 \mathrm{X}$ $+(354.2 \pm$ 33.54. Ihre Abhängigkeit von der Herzfrequenz $X$ war damit größer als bei $Q-A_{0}$.

Da das CTG nur in begrenztem Ausmaß die fetalen Reaktionen auf den Geburtsverlauf wiedergibt und die Feten zudem ganz unterschiedliche Reaktionsmuster und Kompensationsmechanismen zeigen, diskutierten wir die Notwendigkeit einer mehrgleisigen Fetalüberwachung und betonen nachdrücklich ihren Wert gerade bei Risikoschwangerschaften. Erst ein kontinuierliches Monitoring der Sauerstoffversorgung des fetalen Gewebes und die mechanocardiographische Analyse der fetalen Herztätigkeit im Zusammenhang mit biochemischen Blutanalysen lassen eine exakte Diagnose des fetalen Zustandes in utero aussichtsreich erscheinen.

Schliisselwörter: Azidose, Anoxie, Biochemie des Blutes, Blutgaswerte, Cardiotokographie, Elektrocardiographie, Fet, fetale Überwachung, Mechanocardiographie, Polarographie. 


\section{Résumé}

Enregistrement foetal multiphasique dans les grossesses à haut risque.

Le présente recherche a eu pour objet de surveiller le foetus lors de sa délivrance par un nombre de paramètres multiphasiques biochimiques et biophysiques, tels que disponibilité de l'oxygène tissulaire foetal, valeurs quantitatives cardiotocographiques, valeurs biochimiques $\mathrm{du}$ sang materno-foetal, électrocardiogramme (ecg.), mécanocardiogramme (mcg.), etc., pour le diagnostic précis du distress foetal. Un total de 125 grossesses à haut risque ont été examinées. Les électrodes polarographieques montées sur les électrodes de l'ecg. foetal spiral, ont été insérées dans la partie se présentant du foetus au premier stade de délivrance. Les enregistrements cardiotocographiques directs ont été analysés à l'aide des mesures quantitatives dessinées par d'autres. Le mécanocardiogramme foetal a été obtenu par l'ecg., pcg. et les transducteurs ultrasonores, et a été stocké pour l'analyse. Des analyses du gaz du sang et de l'équilibre acido-basique ont été effectuées sur du sang maternal, foetal, ombilical et néonatal, ainsi que des analyses enzymatiques des substrats métaboliques. Les résultats obtenus sont les suivants: (1) Grâce à la surveillance continue de la disponibilité de l'oxygène tissulaire foetal, $\mathrm{l}^{\prime} \mathrm{aO}_{2}$ tissulaire foetal a été considéré comme un bon index pour comprendre les changements dans le ctg. et la physiologie cardio-respiratoire foetale. Bien qu'aucune baisse notable dans $\mathrm{l}^{\prime} \mathrm{O}_{2}$ n'ait été observée dans des délivrance optimales non-stressées, une baisse assez forte dans $\mathrm{l}^{\mathrm{a}} \mathrm{O}_{2}$ d'une durée de plus de 20 à 30 minutes dans des délivrances compliquées a donné des valeurs anormales dans les paramètres cardiotocographiques, d'APGAR et biochimiques sangins artériels ombilicals (Tab' I)'

(2) Les scores d.APGAR sont en corrélation significative avec les valeurs quantitatives cardiotocographiques, telles que bradycardie résiduelle, index de SCHIFRIN-
DAME, index de TIPTON-SHELLEY, aire d'inclinaison, index de KOMAROMY et témps de retard, et aussi avec les valeurs biochimiques sanguines artérielles ombilicales, telles que $\left[\mathrm{H}^{+}\right]$, déficit de base, $\mathrm{P}$ ' inorganique, base de tampon, et concentrations du glycérole (Tab. II). Toutefois, de meilleures corrélations ont été trouvées entre les scores d'APGAR et les profils biochimiques du sang de 15 minutes après la naissance, consitant en $\mid \mathrm{H}^{+}{ }^{+}$, lactate, $\Delta \mathrm{XL}_{\mathbf{f}-\mathrm{m}}, \mathrm{PCO}_{2}, \mathrm{P}$. inorganique, rapport $\mathrm{L} / \mathrm{P},\left[\mathrm{HCO}_{3}^{-}\right]$, concentrations du glycérole et du glucose (Tab. III), suggçrant des facteurs du temps dans ces paramétres biochimiques'

(3) Dans le mcg. foetal, l'étendue normale dans le temps de pré-éjection foetale $\left(\mathrm{Q}-\mathrm{A}_{0}\right)$ a été calculée à $\mathrm{Y}(\mathrm{msec})$ $《-0.132 \mathrm{X}+(89.87 \pm 13.85)$ à FHR $(\mathrm{X})$. Quand l'incidence des anomalies de $\mathbf{Q}-\mathbf{A}_{0}$, soit le raccourcissement, soit la prolongation des étendues citées ci-dessus, dans un temps d'unité a dépassé 20 à $30 \%$ au stade terminal de la délivrance, des valeurs anormales ont été observée dans les paramètres cardiotocographiques quantitatif d'APGAR et biochimiques sanguins (Tab. V). Le systole électromécanique total $\left(\mathrm{Q}-\mathrm{S}_{2}\right)$ a été calculé, à $Y$ 《 -0 '851 $\mathrm{X}+(354.2 \pm(33.54)$, qui a été plus dépendant de FHR que le $\mathrm{Q}-\mathrm{A}_{0}$.

Etant donné que le cardiotocographe n'est qu'un paramètre des réactions foetales au stress de la délivrance et que chaque foetus possède un degré différent de capacités de réserve et de mécanismes de défense contre le stress, on a souligné et discuté la nécessité de la surveillance foetale multiphasique dans les délivrances à haut risque. L'enregistrement continu du niveau de l'oxygène tissulaire foetal et l'analyse mécanocardiographique du coeur foetal et l'analyse mécanocardiographique du coeur foetal, ainsi que les mesures biochimique sanguines pourraient être d.une grande utilité pour le diagnostic précis du bienêtre du foetus dans l'utérus.

Mots-clés: Acidose, anoxie, biochimie sanguine, cardiotocographie, électrocardiographie, enregistrement foetal, foetus, gaz du sang, mécanocardiographie, polarographie.

\section{Bibliography}

[1] Althabe, O., R. L. SChWarcz, S. V. Pose, L. ESCARCENA, R. CALDEYRO-BARCIA: Effects on fetal heart rate and fetal $\mathrm{pO}_{2}$ of oxygen administration to the mother. Amer. J. Obstet. Gynec. 98 (1967) 858

[2] APGAR, V.: Proporsal for new method of evaluation of newborn infant. Current Res. Anesth. Analg. (Cleveland) 32 (1953) 260

[3] BERGMEYER, U., ed.: Methods of enzymatic analysis. 2nd Ed. Academic Press, New York, 1974

[4] FRAN KE, R., H. BELLEE, V. SCHÖNJAHN: Sauerstoffpartialdruckmessung am Feten unter der Geburt. Dtsch. Ges.wesen 26 (1971) 1693

[5] GOODLIN, R. C., E. W. LOWE: Multiphasic fetal monitoring. Amer. J. Obstet. Gynec. 119 (1974) 341
[6] Goodwin, J. W., J. T. DUNNE, B. W. THOMAS: Antepartum identification of the fetus at risk. Can. Med. Assc. J. 101 (1969) 389

[7] HOBEL, C. J., M. A. HYVARINEN, D. M. OKADA, W. OH: Prenatal and intrapartum high-risk screening. I. Predication of the high-risk neonate. Amer. J. Obstet. Gynec. 117 (1973) 1

[8] HUCH, A., R. HUCH: Klinische und physiologische Aspekte der transcutanen Sauerstoffdruckmessung in der Perinatalmedizin. Z. Geburtsh. u. Perinat. 179 (1975) 235

[9] KANEOKA, T.: Electrocardiographic and polarographic observations in the canine fetus. J. Jpn. Obstet. Gynec. Soc. 10 (1963) 19 
[10] KRAUSE, W., G. THIEME, H. VOLKMER: Untersuchungen zur rechenautomatischen Auswertung gasanalytisch und elektronish gewonnener Parameter sub partu. Z. Geburtsh. u. Perinat. 177 (1973) 129

[11] MURATA, Y., H. TAKEMURA, K. KURACHI: Observation of fetal cardiac motion by M-mode ultrasonic cardiography. Amer. J. Obstet. Gynec. 111 (1971) 287

[12] MYERS, G. G., A. J. KRAPOHL, R. D. PETERSON, R. CALDEYRO-BARCIA: New method for measuring lag time between human uterine contraction and the effect on fetal heart rate. Amer. J. Obstet. Gynec. 112 (1972) 39

[13] ORGAN, L. W., A. Bernstein, K. C. SMITH, I. H. ROWE: The pre-ejection period of the fetal heart: Patterns of change during labor. Amer. J. Obstet. Gynec. 120 (1974) 49

[14] SCHIFRIN, B. S., L. DAME: Fetal heart rate patterns. Prediction of APGAR score. J.A.M.A. 219 (1322) 1972
[15] SHELLEY, T., R. H. TIPTON: Dip area, a quantitative measure of fetal heart rate patterns. J. Obstet. Gynaec. Brit. Cwlth. 78 (1971) 694

[16] SUREAU, C., J. Chavinie, B. Michelon, J. P. FELDMAN, M. CANNON, R. LEHOUEZEC: Le problèmme d'interprétation-solutions technique. Gynéc. Obstét. (Paris) 69 (1970) 259

[17] TIPTON, R. H., T. SHELLEY: An index of fetal welfare in labour. J. Obstet. Gynaec. Brit. Cwlth. 78 (1971) 702

[18] WALKER, A., L. PHILliP, L. POWE, C. WOOD: A new instrument for the measurement of tissue $\mathrm{pO}_{2}$ of human fetal scalp. Amer. J. Obstet. Gynec. 100 (1968) 63

[19] WALKER, A., E. DAY, J. TALBOT, C. WOOD: Fetal scalp tissue oxygen tension measurements in relation to maternal dermal oxygen tension and fetal heart rate. J. Obstet. Gynaec. Brit. Cwlth. 78 (1971) 1

Received October 21, 1977, Revised February 13, 1979, Accepted April 30, 1979.
Dr. Tsuyoshi Kaneoka Dept. Obs. Gynec. Fukuoka University School of Medicine 34 Nanakuma, Nishiku, Fukuoka, 814, Japan 